\title{
Inter-Landau Level Scattering Processes in Magnetic Field Assisted THz Quantum Cascade Laser
}

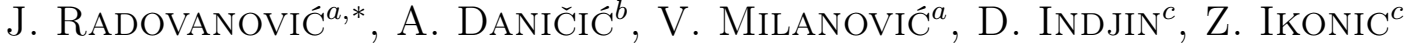 \\ ${ }^{a}$ School of Electrical Engineering, University of Belgrade \\ Bulevar kralja Aleksandra 73, 11120 Belgrade, Serbia \\ ${ }^{b}$ Vinča Institute of Nuclear Sciences, P.O. Box 522, 11001 Belgrade, Serbia \\ ${ }^{c}$ Institute of Microwaves and Photonics, School of Electronic and Electrical Engineering, University of Leeds \\ Woodhouse Lane, Leeds LS2 9JT, UK
}

We present a detailed analysis of GaAs/AlGaAs terahertz quantum cascade laser in the presence of an intense external magnetic field. One of the objectives in further development of $\mathrm{THz}$ quantum cascade laser is the realization of structures operating at higher temperatures. This is difficult to obtain as the operating photon emission energy is smaller than the longitudinal-optical phonon energy in the semiconductor material. With increased temperature, electrons in the upper radiative state gain sufficient in-plane energy to emit an longitudinal-optical phonon, which represents a non-radiative scattering and reduces the optical gain. By applying strong magnetic field, two-dimensional continuous energy subbands become split into series of discrete Landau levels, and at particular values of $B$ it is possible to quench these non-radiative channels. Numerical simulations are performed on two-well design quantum cascade laser operating at $4.6 \mathrm{THz}$, implemented in $\mathrm{GaAs}_{\mathrm{Al}} \mathrm{Al}_{0.15} \mathrm{Ga}_{0.85} \mathrm{As}_{\text {, }}$ and the magnetic field is perpendicular to the epitaxial layers. Strong oscillations of carrier lifetimes for the upper state of the laser transition, as a function of magnetic field are observed, which can be attributed to interface roughness scattering and longitudinal-optical phonon scattering between Landau levels.

PACS: 72.10.--d, 73.21.Fg

\section{Introduction}

In recent years, a lot of effort has been put into developing quantum cascade lasers which emit in the $\mathrm{THz}$ part of the spectrum [1-7]. This range of frequencies is of particular interest for its prospective applications in noninvasive medical diagnostics, chemical sensing, security scanning, telecommunications, etc. However, the impressive performances obtained by quantum cascade lasers (QCL)s in the mid-infrared spectral region, in terms of operating temperature, output power and tunability, cannot easily be transferred to longer wavelengths. The performances of THz QCLs deteriorate rapidly with temperature due to detrimental thermal activation of nonradiative losses [8], so cryogenic cooling is still required for their operation. The highest operating temperature of $225 \mathrm{~K}$ is reported for resonant-phonon design scheme assisted by external magnetic field for additional carrier confinement [3]. The role of the magnetic field is to suppress non-radiative intersubband relaxation processes and thus increase the optical gain [1, 3, 8-18]. Hence, detailed understanding of various scattering mechanisms,

\footnotetext{
* corresponding author; e-mail: radovanovic@etf.bg.ac.rs
}

relevant for laser operation, may be an important factor in improving its performance.

In this paper we study electron relaxation rates for the upper state of the laser transition, due to electronlongitudinal-optical (LO) phonon interactions and interface roughness scattering, for a structure subjected to a quantizing magnetic field parallel to the confinement direction. The QCL under consideration comprises only two quantum wells per period and is intended for operation at $4.6 \mathrm{THz}$ [2]. Owing to the simplicity of this design, only three subbands per period are mainly involved in electron transport which facilitates studying of carrier relaxation processes. We find pronounced oscillations of the scattering rates with magnetic field and confirm that elastic scattering due to interface roughness is an important relaxation mechanism at lower temperatures, in particular when LO-phonon emission is suppressed.

\section{Theoretical considerations}

We analyze the simplest (two-well) design of $\mathrm{THz}$ QCL [2], whose conduction band profile is illustrated in Fig. 1. The lower laser level is depopulated via intrawell LO-phonon scattering, in order to maintain a short lifetime for this level, while radiative transition between subbands denoted by $n=3$ and $n=2$ is diagonal. The 
injection of carriers into the active region and extraction from the lower subband is achieved via resonant tunneling. In the absence of an external magnetic field, the electronic subbands from Fig. 1 have free particle-like energy dispersion in the direction parallel to the QW planes: $E_{n}\left(k_{\|}\right)=E_{n 0}+\hbar^{2} k_{\| \mid}^{2} / 2 m_{\| n}\left(E_{n 0}\right)$, where $m_{\| n}\left(E_{n 0}\right)$ is the energy-dependent in-plane effective mass, and $k_{\|}$is the in-plane wave vector. As the operating temperature increases, electrons in the upper radiative state gain sufficient in-plane energy to emit an LO-phonon, which results in a fast thermally activated scattering process and a reduction in optical gain. However, when the structure from Fig. 1 is subjected to a strong magnetic field $B$ in the $z$-direction, continuous subbands $E_{n}\left(k_{\|}\right)$transform into series of individual (strictly discrete) states at energies $E_{n, l} \approx E_{n 0}+(l+1 / 2) \hbar \omega_{c}$, where $l=0,1,2, \ldots$ is the Landau index and $\omega_{c}=e B / m^{*}$ is the cyclotron frequency. The values of $B$ which give rise to resonant LO phonon emission are found by solving equation: $E_{3,0}-E_{n, l}=\hbar \omega_{\mathrm{LO}}(n$ corresponds to lower subbands $)$, where $\hbar \omega_{\text {LO }}$ is the LO phonon energy.

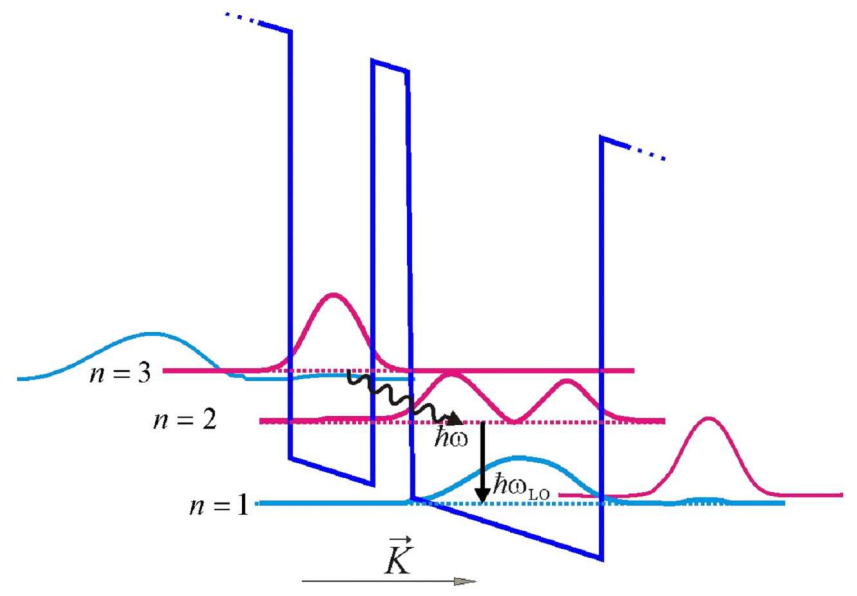

Fig. 1. A schematic diagram of a single period of twowell $\mathrm{THz}$ quantum cascade laser [2]. Electrons are injected from preceding period into subband $n=3$ by resonant tunneling. The optical transition occurs between subbands $n=3$ and $n=2$, while the depopulation of the lower laser subband $n=2$ relies on fast intrawell electron-LO phonon scattering into subband $n=1$ (the injection subband for the following period).

The electron-LO phonon scattering rates on transitions between the initial state $E_{i}=E_{n_{i}, l_{i}}$ and the final state $E_{f}=E_{n_{f}, l_{f}}$ may be found by following the approach presented in Ref. 17:

$$
\begin{aligned}
& \frac{1}{\tau_{n_{i}, l_{i} \rightarrow n_{f}, l_{f}}^{\mathrm{LO}}}=\frac{\mathrm{e}^{2} \omega_{\mathrm{LO}}}{4 \varepsilon_{0} \varepsilon_{p}} \frac{1}{\delta \sqrt{2 \pi}} \mathrm{e}^{-\frac{\left(E_{n_{i}, l_{i}}-E_{n_{f}, l_{f}}-\hbar \omega_{\mathrm{LO}}\right)}{2 \delta^{2}}} \\
& \quad \times\left(n_{q}+1\right) \int_{0}^{\infty}\left|F\left(q_{||}\right)\right|^{2} G\left(q_{\|}\right) \mathrm{d} q_{\|},
\end{aligned}
$$

where $\varepsilon_{p}^{-1}=\varepsilon_{\infty}^{-1}-\varepsilon_{s}^{-1}\left(\varepsilon_{\infty}\right.$ and $\varepsilon_{s}$ are the static and high-frequency relative dielectric constant, respectively), $\delta$ is the width of the Gaussian distribution of energy difference $E_{n_{i}, l_{i}}-E_{n_{f}, l_{f}}$ and $n_{q}=\left[\exp \left(\hbar \omega_{\mathrm{LO}} / k_{\mathrm{B}} T\right)-1\right]^{-1}$ is the mean number of LO phonons. Furthermore, $q_{\|}$ is the in-plane component of the phonon wave-vector $\boldsymbol{q}=\left(q_{z}, q_{\|}\right)$and $F\left(q_{||}\right)$is the overlap integral which reads

$$
\begin{aligned}
& \left|F\left(q_{\|}, l_{i}, l_{f}\right)\right|^{2}=\mathrm{e}^{-\frac{q_{\|}^{2}}{2 \beta^{2}}} \frac{l_{i} !}{l_{f} !}\left(\frac{q_{\|}^{2}}{2 \beta}\right)^{l_{f}-l_{i}} \\
& \quad \times\left[L_{l_{i}}^{l_{f}-l_{i}}\left(q_{\|}^{2} / 2 \beta\right)\right]^{2},
\end{aligned}
$$

where $\beta=\sqrt{e B / \hbar}$ and $L_{m}^{k}(x)$ represents the associated Laguerre polynomial. Finally, $G\left(q_{\|}\right)$stands for the form factor given by:

$$
\begin{aligned}
& G\left(q_{\|}\right)= \\
& \quad \iint \eta_{i}^{*}(z) \eta_{f}(z) \eta_{i}\left(z^{\prime}\right) \eta_{f}^{*}\left(z^{\prime}\right) \mathrm{e}^{-q_{\|}\left|z-z^{\prime}\right|} \mathrm{d} z \mathrm{~d} z^{\prime},
\end{aligned}
$$

where $\eta_{i}$ and $\eta_{f}$ denote the $z$-dependent parts of the electronic wavefunctions.

To evaluate the interface roughness scattering rate we start by defining the perturbation Hamiltonian [13, 19]:

$$
\hat{H}_{I R}^{\prime}=U_{0} \delta\left(z-z_{i}\right),
$$

where $U_{0}$ is the barrier height, $z_{i}$ is the interface position and $\delta$-function underlines the local character of this interaction. We assume that the spatial distribution of roughness may be chosen to follow the Gaussian correlation function $[13,19]$

$$
\left\langle\Delta(\boldsymbol{r}) \Delta\left(\boldsymbol{r}^{\prime}\right)\right\rangle=\Delta^{2} \mathrm{e}^{-\frac{\left|\boldsymbol{r}-\boldsymbol{r}^{\prime}\right|^{2}}{\Lambda^{2}}},
$$

where $\Delta$ is the mean height of the roughness and $\Lambda$ is the correlation length. The scattering matrix element can therefore be expressed as:

$$
M_{i, f}=\int_{V} \Psi_{n_{i}, l_{i}, k_{x_{i}}}^{*} \hat{H}_{I R}^{\prime} \Psi_{n_{f}, l_{f}, k_{x_{f}}} \mathrm{~d} V,
$$

where $\hat{H}_{I R}^{\prime}$ is given by Eq. (4). The wavefunctions $\Psi_{n, l, k_{x}}(x, y, z)$ represent a product of three functions, $\eta_{n}(z), \Phi_{l, k_{x}}(x, y)$ and $\exp \left(i k_{x} x\right) / \sqrt{L_{x}}$ ( $L_{x}$ is the halflength of the structure in $x$-direction, $\left.L_{x} \rightarrow \infty\right)$. By inserting these wavefunctions into Eq. (6), we obtaind the averaged matrix element squared:

$$
\begin{aligned}
& \left\langle\left|M_{i, f}\right|^{2}\right\rangle=\frac{\left|F_{i f}\right|^{2} \Delta^{2} \Lambda \sqrt{\pi} \alpha}{L_{x}} \mathrm{e}^{-\frac{\Delta k_{x}^{2}}{2}\left[\frac{2+\Lambda^{2} \beta^{2}}{2 \beta^{2}}\right]} \\
& \quad \times \zeta\left(\Delta k_{x}\right) .
\end{aligned}
$$

Here, $\Delta k_{x}=k_{x_{i}}-k_{x_{f}}, \quad F_{i f}=U_{0} \eta_{i}^{*}\left(z_{i}\right) \eta_{f}\left(z_{i}\right)$ and $\alpha=\beta^{2} /\left(\pi l_{f} ! l_{i} ! 2^{l_{f}+l_{i}}\right)$. The form factor $\zeta\left(\Delta k_{x}\right)$ is given by:

$$
\begin{aligned}
& \zeta\left(\Delta k_{x}\right)=\iint \exp \left(-\beta^{2}\left[t^{2}+(t-w)^{2}\right]-\frac{w^{2}}{\Lambda^{2}}\right) \\
& \times H_{l_{i}}\left[\beta t-\frac{\Delta k_{x}}{2 \beta}\right] H_{l_{i}}\left[\beta(t-w)-\frac{\Delta k_{x}}{2 \beta}\right] \\
& \times H_{l_{f}}\left[\beta t+\frac{\Delta k_{x}}{2 \beta}\right] H_{l_{f}}\left[\beta(t-w)+\frac{\Delta k_{x}}{2 \beta}\right] \mathrm{d} t \mathrm{~d} w
\end{aligned}
$$


tering rate can now be expressed as [17]:

$$
\begin{aligned}
& \frac{1}{\tau_{n_{i}, l_{i} \rightarrow n_{f}, l_{f}}^{\mathrm{IR}}}=\frac{\sqrt{2 \pi}}{\hbar \sigma} \mathrm{e}^{\frac{\left(E_{n_{i}, l_{i}}-E_{n_{f}, l_{f}}\right)^{2}}{2 \delta^{2}}} \\
& \quad \times \sum_{k_{x_{i}}, k_{x_{f}}}\left\langle\left|M_{i, f}\right|^{2}\right\rangle .
\end{aligned}
$$

Clearly, $\left\langle\left|M_{i, f}\right|^{2}\right\rangle$ depends only on the difference between $k_{x_{i}}$ and $k_{x_{f}}$, not on their individual values, hence by averaging over $k_{x_{i}}$ in the above expression, we obtain $\sum_{k_{x_{i}}, k_{x_{f}}}\left\langle\left|M_{i, f}\right|^{2}\right\rangle \rightarrow \frac{1}{\pi} \int_{-\infty}^{\infty}\left|M_{i, f}\right|^{2} d\left(\Delta k_{x}\right)$.

\section{Numerical calculations}

The GaAs $/ \mathrm{Al}_{0.15} \mathrm{Ga}_{0.85} \mathrm{As}$ QCL structure under consideration, consists of two quantum wells per period, and is designed to emit radiation at $4.6 \mathrm{THz}$ [2]. Fig. 2 illustrates the conduction band diagram of the structure including one central and two adjacent periods with relevant wavefunctions squared. The energy difference between subbands 3 and 2 is found to be $19 \mathrm{meV}$, while $E_{2}-E_{1}=36 \mathrm{meV} \approx \hbar \omega_{\mathrm{LO}}$. The layer thicknesses starting from the injector barrier read: 56/71/31/167 $\AA$.

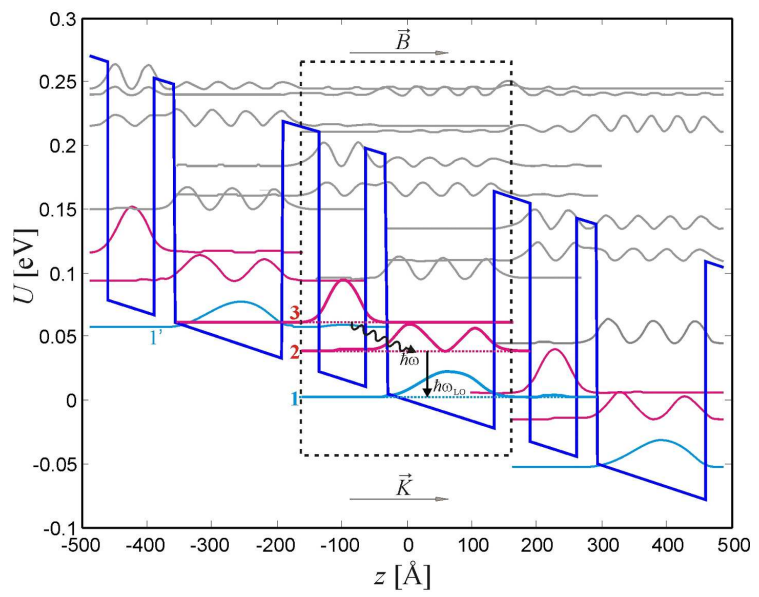

Fig. 2. Conduction band profile of a $\mathrm{THz}$ quantum cascade laser. Three periods of the structure are displayed, and the central one is outlined by a dashed rectangle. The layer thicknesses starting from the injector barrier are $56 / 71 / 31 / 167 \AA$, and the electric field is $K=17 \mathrm{kV} / \mathrm{cm}$.

Numerical parameters used in calculation are: $m^{*}=$ $0.0665 m_{0}\left(m_{0}\right.$ is the free electron mass $)$, the conduction band discontinuity between GaAs and AlAs is $\Delta E_{c}=$ $0.8355 \mathrm{eV}, \Delta=1.5 \AA, \Lambda=60 \AA, \delta=6 \mathrm{meV}, \varepsilon_{\infty}=10.67$, $\varepsilon_{s}=12.51, T=77 \mathrm{~K}$. Clearly, it is quite a complex task to evaluate the appropriate values of parameters $\Delta$ and $\Lambda$ which describe the interface roughness scattering, given that they depend on the QCL growth technique as well. Moreover, as pointed out in Ref. 19, in case of a quantum well (without magnetic field), the interface roughness (IR) scattering rates differ for an full order of magnitude when correlation length $\Lambda$ changes from $10 \AA$ to $100 \AA$. We have chosen the value of $60 \AA$, which is very close to the typical values for GaAs/AlGaAs interface [19]. As for the influence of mean height of roughness $\Delta$, the IR relaxation rate is directly proportional to $\Delta^{2}$, so it can easily be rescaled for other values of $\Delta$ reported in the literature, e.g. $\Delta=3 \AA$.

When the structure from Fig. 2 is placed in a strong external magnetic field in the $z$-direction, the $2 \mathrm{D}$ subbands $E_{n 0}+\hbar^{2} k_{\| \mid}^{2} / 2 m_{\| n}\left(E_{n 0}\right)$ split into series of discrete LLs, the energies of which (with band nonparabolicity included) are given by [20]: $\quad E_{n, l}=E_{n 0}+(l+1 / 2) \hbar e B / m_{\| n}\left(E_{n 0}\right)-$ $1 / 8\left[\left(8 l^{2}+8 l+5\right) \alpha_{1}^{\prime}+\left(l^{2}+l+1\right) \beta_{1}^{\prime}\right]\left(\hbar e B / m^{*}\right)^{2}$. The in-plane electron effective mass is here calculated as $m_{\|}\left(E_{n 0}\right)=m^{*}\left[1+\left(2 \alpha_{1}^{\prime}+\beta_{1}^{\prime}\right) E_{n 0}\right][14,15]$, where the nonparabolicity parameters $\alpha_{1}^{\prime}$ and $\beta_{1}^{\prime}$ are evaluated according to Ref. 20. The total relaxation rate for transitions from the ground LL of the third subband into the sets of LLs of all relevant lower subbands (of the central and surrounding periods), due to LO phonon scattering and interface roughness scattering, is displayed in Fig. 3, for magnetic fields in the range $0.5-18 \mathrm{~T}$.

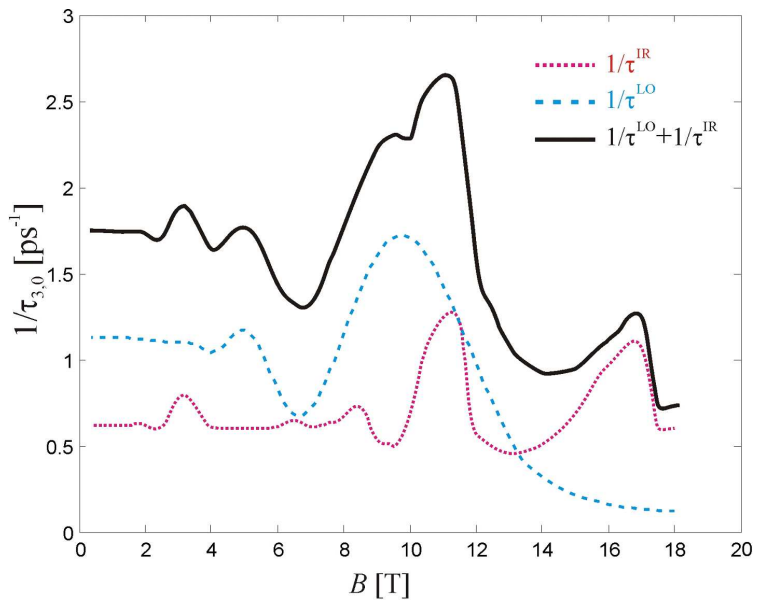

Fig. 3. The total electron relaxation rate due to emission of LO phonons and interface roughness scattering as a function of the magnetic field, for transitions from the state 3,0 into Landau levels belonging to lower subbands, at $T=77 \mathrm{~K}$.

From Fig. 3, we can see that oscillations of the relaxation rate with $B$ are very pronounced, with several distinct peaks. The first set of maxima occurs at values of the magnetic field which make the resonance conditions for LO phonon emission fulfilled. Conversely, when the arrangement of LLs is such that there is no level situated at $\approx \hbar \omega_{\text {LO }}$ below the state 3,0 , this type of scattering is inhibited, and therefore the corresponding relaxation rate is decreased. On the other hand, when $E_{3,0} \approx E_{n, l}$ (with $n$ corresponding to all relevant lower subbands from Fig. 2), the additional relaxation path is opened for electrons to elastically scatter into en- 
ergetically nearby levels. These increases in relaxation rate are assigned to interface roughness scattering mechanism. However, the relative influence of elastic and inelastic scattering on output properties of QCL, namely on the optical gain, needs also to be checked out by solving a full set of rate equations, which describe the electron distribution over all the states in the system, as described in Refs. 14-16. As pointed out in Ref. 7, the influence of the ground state of the thicker well (1' in Fig. 2) must be taken into account when calculating the optical gain, as it extends into the thinner well of the next active region at energy very close to that of state 3 . In addition, it may be useful to perform calculations of scattering rates at higher temperatures as well. In our model, the temperature dependent quantity is $n_{q}+1=\exp \left(\hbar \omega_{\mathrm{LO}} / k_{\mathrm{B}} T\right) /\left[\exp \left(\hbar \omega_{\mathrm{LO}} / k_{\mathrm{B}} T\right)-1\right]$ in Eq. (1). At low operating temperature, it is very close to unity (namely, 1.0012 and 1.032 at $77 \mathrm{~K}$ and $120 \mathrm{~K}$, respectively), while it increases significantly at room temperature $(1.33$ at $300 \mathrm{~K})$. Evidently, with rising $T$ the relaxation rate $1 / \tau^{\mathrm{LO}}$ is increased, while $1 / \tau^{\mathrm{IR}}$ remains unchanged, therefore the total relaxation rate becomes increased. Furthermore, for high enough electron surface densities, the potential profile must be found selfconsistently, hence all the electron energies and wavefunction would become temperature dependent, and the influence of temperature in both $1 / \tau^{\mathrm{LO}}$ and $1 / \tau^{\mathrm{IR}}$ would become quite complicated. These calculations will be completed within the next stage of our work, with the goal of providing a clearer understanding of the effects of nonradiative scattering processes on the operation of $\mathrm{THz}$ QCLs

\section{Conclusion}

We have presented a detailed model, together with numerical results, for calculating electron relaxation rates for two-well resonant-phonon design based $\mathrm{THz}$ quantum cascade lasers, in strong magnetic fields. In particular, the aim of this work was to obtain the electrons relaxation rates from the upper laser level due to LO phonon scattering (inelastic) and interface roughness scattering (elastic) and compare their relative weights. It is apparent that both relaxation mechanisms must be included in the course of calculating optical gain of the structure. Furthermore, both types of scattering are strongly influenced by magnetic field variations as well, hence by varying the strength of this field, we are able to detect regions with significantly reduced scattering, which is important for efficient laser operation.

\section{Acknowledgments}

This work was supported by the Ministry of Science (Republic of Serbia), ev. no. III.

\section{References}

[1] N. Péré-Laperne, L.A. de Vaulchier, Y. Guldner, G. Bastard, G. Scalari, M. Giovannini, J. Faist, A. Vasanelli, S. Dhillon, C. Sirtori, Appl. Phys. Lett. 91, 062102 (2007).

[2] S. Kumar, C.W.I. Chan, Q. Hu, J. Reno, Appl. Phys. Lett. 9514110 (2009).

[3] A. Wade, G. Fedorov, D. Smirnov, S. Kumar, B.S. Williams, Q. Hu, J.L. Reno, Nature Photon. 3, 41 (2009).

[4] G. Scalari, C. Walther, L. Sirigu, M.L. Sadowski, H. Beere, D. Ritchie, Phys. Rev. B 76, 115305 (2007).

[5] M.I. Amanti, G. Scalari, R. Terazzi, M. Fischer, M. Beck, J. Faist, A. Rudra, P. Gallo, E. Kapon, New J. Phys. 11, 125022 (2009).

[6] G. Scalari, C. Walther, M. Fischer, R. Terazzi, H. Beere, D. Ritchie, J. Faist, Laser Photon. Rev. 3, 45 (2009).

[7] G. Scalari, M.I. Amanti, C. Walther, R. Terazzi, M. Beck, J. Faist, Opt. Express 18, 8043 (2010).

[8] I. Savić, N. Vukmirović, Z. Ikonić, D. Indjin, R.W. Kelsall, P. Harrison, V. Milanović, Phys. Rev. $B$ 76, 165310 (2007).

[9] I. Savić, Z. Ikonić, N. Vukmirović, D. Indjin, P. Harrison, V. Milanović, Appl. Phys. Lett. 89, 011109 (2006).

[10] D. Indjin, Z. Ikonić, V.D. Jovanović, N. Vukmirović, P. Harrison, R.W. Kelsall, Semicond. Sci. Technol. 20, S237 (2005).

[11] I. Savić, Z. Ikonić, V. Milanović, N. Vukmirović, V. D. Jovanović, D. Indjin, P. Harrison, Phys. Rev. $B$ 73, 075321 (2006).

[12] Y. Chen, N. Regnault, R. Ferreira, B.-F. Zhu, G. Bastard, Phys. Rev. B 79, 235314 (2009).

[13] A. Leuliet, A. Vasanelli, A. Wade, G. Fedorov, D. Smirnov, G. Bastard, C. Sirtori, Phys. Rev. B 73, 085311 (2006).

[14] J. Radovanović, V. Milanović, Z. Ikonić, D. Indjin, P. Harrison, J. Appl. Phys. 97, 103109 (2005).

[15] J. Radovanović, A. Mirčetić, V. Milanović, Z. Ikonić, D. Indjin, P. Harrison, R.W. Kelsall, Semicon. Sci. Technol. 21, 215 (2006).

[16] A. Daničić, J. Radovanović, V. Milanović, D. Indjin, Z. Ikonić, J. Phys. D: Appl. Phys. 43, 045101 (2010).

[17] C. Becker, A. Vasanelli, C. Sirtori, G. Bastard, Phys. Rev. B 69, 115328 (2004).

[18] C. Becker, C. Sirtori, O. Drachenko, V. Rylkov, D. Smirnov, J. Leotin, Appl. Phys. Lett. 81, 16 (2002).

[19] T. Unuma, M. Yoshita, T. Noda, H. Sakaki, H. Akiyama, J. Appl. Phys. 93, 3 (2003).

[20] U. Ekenberg, Phys. Rev. B 407714 (1989). 